\title{
A Contemporary Guide to Beam Dynamics
}

\author{
Étienne Forest* and Kohji Hirata ${ }^{\dagger}$ \\ KEK, National Laboratory for High Energy Physics, \\ Tsukuba, Ibaraki 305, Japan
}

\begin{abstract}
A methodological discussion is given for single particle beam dynamics in circular machines. The discussions are introductory, but (or, even therefore) we avoid to rely on too much simplified concepts. We treat things from a very general and fundamental point of view, because this is the easiest and rightest way to teach how to simulate particle motion and how to analyze its results. We give some principles of particle tracking free from theoretical prejudices. We also introduce some transparent methods to deduce the necessary information from the tracking: many of the traditional beam-dynamics concepts can be abstracted from them as approximate quantities which are valid in certain limiting cases.
\end{abstract}

\footnotetext{
- Permanent address, Exploratory Studies Group, Accelerator and Research Division, Lawrence Berkeley Laboratory, 1 Cyclotron Road, MS 71-259, Berkeley, California 94720, USA. Electronic mail: ETIENNE@LBL.Bitnet

${ }^{\dagger}$ Electronic meil: HIRATA@JPNKEKVM.Bitnet 


\section{Contents}

1 Preface $\quad 2$

2 Local System: LEGO Block 5

2.1 Local Coordinates: the Faces of each Block . . . . . . . . . . . . . . . 5

2.2 Local Particle Dynamics: Modeling principles . . . . . . . . . . . 6

2.3 State Variables . . . . . . . . . . . . . . . . . 7

2.4 Connecting Local Blocks into a Ring . . . . . . . . . . . . . . . 9

3 Global System: One-Turn Map $\quad 11$

3.1 Approximations of the One-turn Map: $N$-Jets . . . . . . . . . . 12

3.2 Global Dynamics of $N$-jets: the Paradox . . . . . . . . . . . . . 14

3.3 So, where are my Global Goodies? Answer: Normal Forms . . . . . . . . . 15

3.4 Equilibrium Beam Envelope . . . . . . . . . . . . . . . . 17

3.5 WARNING about Perturbation Theory . . . . . . . . . . . . 19

3.6 The Issue of Useful Formulas . . . . . . . . . . . . . . . . . . 19

4 Summary $\quad 22$

A Fibre Bundle Structure of LEGO Loop

B Rectangular Bend $\quad 25$

\section{Preface}

How to Avoid Reading This Paper If you are so lazy that you do not want to read this report from beginning to end, you can replace it by remembering the following phrases.

1. The one-turn map (plus partial transfer map) contains everything.

2. To construct it, you should not rely on so-called beam-dynamics concepts. Use Lorentz equation only.

3. To use the one-turn map, you can ignore what you did in constructing it. Try to use physical and/or well-defined concepts only.

The Aim of This Paper The authors agree that it is necessary to have a short tutorial paper on beam dynamics of circular machines from a modern point of view. The purpose is to emphasize the modern approach which decouples "simulation" from "analysis". In our view, this is by far the best way to approach the topic of circular rings and quasiperiodic systems. Unfortunately, this paper is not comprehensive enough as a textbook. Instead, we will give a large scale overview of the structure of circular ring dynamics as the authors perceived it. The readers should extract from this paper a general understanding of what we call the "modern" approach and this should serve him or her as a guide into more detailed studies. 
Global and Local Information This paper is based on a fundamental distinction between local and global information. A quantity is called local if it is derivable from the individual magnet irrespective of the maginet position in the ring and even irrespective of the ring itself. For example, the trajectory of a particle through a magnet is local. If a particle suddenly appear at the entrance of a quadrupole, we can predict its future position and momentum without any knowledge of its origin. We simply integrate " $F=m a "$ or Lorentz equation through the magnet. Whether the magnet is installed in a ring or sitting on a testing bench alone, like a prototype, it does not matter. This is intuitively obvious: if you are an electron going through a circular ring you don't need more than "F=ma" to find your way around.

Global information, on the contrary, is derivable only after the full ring is produced. For example, the dynamic aperture has no meaning whatsoever if we cannot iterate the one-turn map (i.e. circulate particles in the machine). Also the "so-cal'ed" closed orbit is a property of the global object. Things like the Courant-Snyder invariants appears at this stage as invariants of the linear part of the one-turn map.

Notice that all the local properties are controlled by the underlying equation of motion " $F=m a$ " irrespective of purpose. The global properties are direct consequences of the purpose of the ring: to iterate or circulate a beam for many turns. The global properties emerged from our feeble attempt to interpret what we see. They are part of the interpretation of the one turn map. The phenomenological and the complex concepts of periodic systems (tunes, beta functions etc...) come in at the end when one interprets the results. To clarify further, we give a few examples:

\begin{tabular}{c|c} 
Local & Global \\
simulation of the one-magnet map & interpretation of the one-turn map \\
\hline reference coordinate & closed orbit \\
deviation from nominal orbit & betatron amplitude \\
magnetic field & betatron function \\
beam envelope & equilibrium emittance \\
amount of synchrotron radiation & damping partition number
\end{tabular}

The Modern Approach The modern approach consists in separating the creation of the one-turn map which is a local endeavour from its subseçuent analysis which is a global problem. This separation is done at all levels: computational and conceptual.

In this paper, we stress that the construction of a tracking code is entirely a local problem. Each magnet is defined by a local Hamiltonian whose frame of reference is determined by purely local geometrical considerations (shape and symmetry of the magnetic field). For example, the particular Frenet-Serret curve used is not necessarily a trajectory produced by the magnet. Our only guiding principle in choosing a frame of reference is how to push particles through the magnet in a way as simple as possible, given some complex magnetic field. What the rest of the ring does is (and must be) of no concern to us in this process. Of course, if the individual magnets have conflicting geometries, we must learn how to patch them together. This is the LEGO $^{1}$ block approach and we

\footnotetext{
${ }^{1}$ LEGOQ is a registered trademark of Interlego, A.G., which is not in any way related to the authors.
} 
discuss it in section 2.

By connecting local systems together, we end up with a ring. Here the analysis begins. How do we extract our beta functions, tune shifts, emittances and all the rest of these "beautiful" concepts? Our technique is mathematically totally disconnected from the construction of the tracking code. How can we do this? We forget everything about the local properties (Hamiltonian of each element) and concentrate on the global one-turn map. In fact, we express standard perturbation theory only in terms of the one-turn map. Where the one-turn map came from and what various frames of reference were used to compute it become totally irrelevant. To illustrate this point, we give a simple example: if you have a $2 \times 2$ matrix representing a ring and I asked you for the tune, you will take the trace and that is the end of it. The matrix is an approximate one-turn map and the tune is readily extractable from it. In general, we express all of perturbation theory in a "Hamiltonian-free" context where the ring is defined as a finite set of maps. This is outlined in section 3 .

In conclusion, we divide the work into two parts:

1. the computation of the one-turn map (local)

2. the analysis of the map (global).

The separation between the local and global concepts is so complete, as we pointed out, that it is possible to teach one independently of the other.

Contrasting with the Classical Approach Traditionally, one begins with a definition of global coordinates according to Frenet-Serret formulas spanned with respect to a somewhat realistic design trajectory. The magnetic field is then expressed in terms of this coordinate system. The resulting Hamiltonian is global.

The traditional approach is an attempt to derive a representation of the equation of motions which is suitable simultaneously to tracking and to the computation of global quantities such as betatron functions $(\beta)$, the tunes, etc. This may be possible in principle but is extremely difficult because of the complexity of a circular ring. For pedagogical purpose, the general problem is usually swept under the carpet and only idealized rings are taught. However, this is not pedagogical: the inexperienced physicist may be gravely mislead if (s)he thinks that this "double purpose" parametrization of the Hamiltonian ( applicable both for tracking and analysis) is always possible. In fact, in general, one cannot find a global and workable Hamiltonian in global and faithful Frenet-Serret variables. The trusting student is lead into a dead end by being introduced to concepts which work only on a certain class of idealized problems (without the horizontal-vertical coupling, without the cavity, without any nonlinear elements, etc.).

Our point is that we must imitate the real world in a simulation code. Our simulation techniques (symplectic integration etc......) may be dictated by global purposes but the code must remain local in computing tracking data and approximating the one-turn map. That is the only way to keep it simple in a non-ideal situation. Trying to choose the Hamiltonian to facilitate the computation of global entities (the phase advance or the tune shifts with amplitude for example) is an absolute dead end. 
We say: compute these quantities in terms of finite time $(=s)$ (typically one-turn) maps if you are serious in your attempt to handle the calculation. Divide a ring into finite number of pieces and obtain maps to connect them. The Hamiltonian-free formalism of perturbation theory will allow you to compute any quantity you desire with this finite number of maps. The theory is based on a global definition of the ring as a finite product of maps. This number of maps is dictated by the purpose of your calculation. Quite often you will need only one map: the one-turn map. The standard Hamiltonian theory, viewed from this angle, forces you to consider an continuously infinite number of maps because the Hamiltonian represents the map for an infinitesimal time $(=s)$ step. The reader should consult the theoretical paper on the topic[6].

An attitude of directly evaluating a one-turn map on one hand and theoretically analyzing its result on the other hand, is more powerful computationally as well as conceptually for the study of circular ring. Of this, we are absolutely certain. Other approaches, if necessary, are easily deduced from it.

So, let us start with the local description: the LEGO blocks.

\section{Local System: LEGO Block}

In this section, we discuss how the local coordinate system attached to each magnets permits us propagate physical (local) quantities across the blocks. In section 2.1 we define the blocks, in 2.2 we state some fundamental principles in the actual construction of the blocks and finally in 2.3 we describe what can be propagated through a block. The connection of the blocks is done in 2.4 .

\subsection{Local Coordinates: the Faces of each Block}

We have the complete freedom to choose the coordinate system. To make the discussion more visual ${ }^{2}$, however, let us compose a ring with only two kinds of blocks, a bend (B) and drift (D). From the point of view of our discussion, these two blocks are defined as follows (see Fig. 1) :

1) the drift is a block which has two parallel faces on which a local Cartesian frame is attached. The frames are identical (same unit vectors) and the line which link them has a length $I$ and is perpendicular to both frames or faces. Notice that any element whose primary purpose is to focus the trajectory in some fashion but not to bend it is a drift for the purpose of this discussion (quadrupole, sextupole, wiggler, solenoid etc....).

2) A bend is a block characterized by two faces with parallel $y$-axes but $x$-axes meeting at an angle $\theta$. The two $x$-axes and the line joining the two origins form a plane perpendicular to the two faces. An arc of circle of length $L$ passes through the origin of both faces perpendicilarly. Such a block has for primary purpose to bend an incoming particle by approximately $\theta$. Notice that nothing is said about the internal details of this magnet (whether it is a sector bend, a bend with irregular field or whatever, is immaterial for the following discussion).

\footnotetext{
${ }^{2}$ For more mathematical and general discussion, see Appendix A.
} 
Figure 1: Fundamental LEGO blocks of the ring. Local coordinate systems can be constructed around them. Bold lines represent the direction of $y$ and dashed $x$. Note that $B$ does not imply the sector bend.
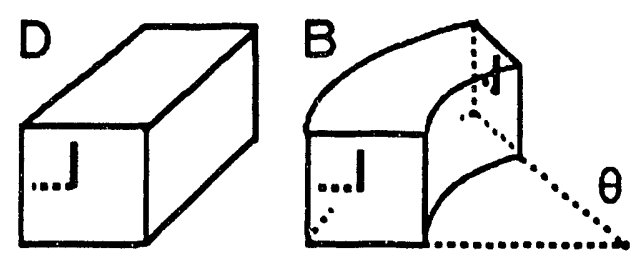

In summary, the Bend is characterized by a bending radius $(\rho)$, (nominal) length $(L)$ and two faces with the proper reference frame. The Drift is characterized by the length $(L)$ and also two properly "framed" faces.

The reader will notice that the drift is a special case of the bend: let $\theta$ go to zero and $\rho$ to infinity while maintaining their product equal to $\mathrm{L}$. In fact the bend is the fundamental block which is used in the composition of bigger blocks.

Note that the LEGO block has little to do with physics in it. It simply states that we are interested in the (deterministic) motion through each block, which is to be represented by a (generally nonlinear) transformation ${ }^{3}$

$$
\boldsymbol{x}_{\text {in }} \longrightarrow \boldsymbol{x}_{\text {out }}=\mathbf{f}\left(\boldsymbol{x}_{\text {in }}\right),
$$

where $\boldsymbol{f}$ is a vector of (generally nonlinear) functions connecting the local coordinate $\boldsymbol{x}_{\text {in }}$ defined at the entrance face and the local coordinate $x_{\text {out }}$ at the exit face. See Fig. 2 . The construction of such a transformation will depend on internal consideration which are not needed to understand the LEGO approach. We will see that in general the LEGO block for a single magnet might be itself composed of several blocks: this internal structure is dictated by magnet geometry and is not necessary at this point of our discussion.

Figure 2: The map from the entrance to the exit of a LEGO block. A block indicates two faces where $\mathbf{x}$ is defined
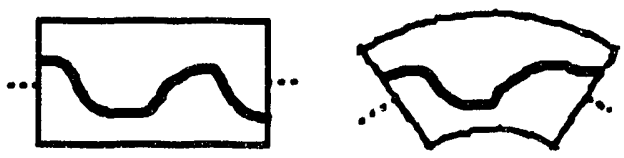
but nothing more.

\subsection{Local Particle Dynamics: Modeling principles}

Modeling Problem For most of the cases, given some equations of motion, it is quite difficult to find the exact trajectory. Therefore you should introduce some approximations, which are accurate enough for your purpose and simple enough to permit calculations within a finite time. The particular form of the map one elects to use is called the

\footnotetext{
${ }^{3}$ We do not use the coordinates explicitly except for Appendix B. For the sake of definiteness, however, we say $x$ in our mind is $\left(x, p_{x}, y, p_{y}, t,-E\right)$. Here $x$ and $y$ are understood; $p_{x}$ and $p_{y}$ are the projection of the relativistic 3-momentum $\mathbf{p}$ to $x$ and $y$ direction: $p_{x}=\mathbf{p} \cdot \mathbf{e}_{x}$, etc. Here $\mathbf{e}_{x}$ is the unit vector in the direction of $x$. The $t$ is the time and $E$ is the energy.
} 
model. The element is defined by a block ( 2 frames of reference) and the model (how to propagate a particle from one face to the other). We thus say

$$
\text { element }=\text { block }+ \text { model }
$$

The model defines a map within an element: it should transform the state variables from the entrance to the exit as Eq.(1). For example the block we called the "bend" in section 2.1 can, for some purposes, be a simple drift, keeping the transverse momenta invariant and changing only the positions. Or it can be itself a complex composition of blocks describing the body and the fringe field region. It can also have non-Hamiltonian effects such as radiation etc...The list is endless, but again it is immaterial for stating our modeling principles. In general, an element can be defined also by a certain number of blocks (if it is a multi-block element) and by a model for each block, the equation of motion in each block with its integration method and its associated number of integration steps. Now we state two central principles:

Principle 1 (Locality) The 6-vector $x_{\text {out }}$ is written only in terms of $x_{\text {in }}$ and the local parameters intrinsic to that element. It applies in the same way regardless of the particle passing only once or many times through the element.

This principle rejects codes written with some global Frenet-Serret variables. It also rejects codes which require the knowledge of the closed orbit or the lattice functions in order to perform tracking. Implicitly, it also affirms that any coordinate system can be used internally as long as they correctly relate $\boldsymbol{x}_{\text {in }}$ and $\boldsymbol{x}_{\text {out }}$.

Principle 2 (Permanence) Once you choose the blocks and their model, you stick to them during the entire calculation. The model can be changed only at the beginning or end of a calculation if it needs to be simplified or complexified. It must never be changed during the calculation.

For example, some numerical analysis techniques employ a "variable time step" integrator. This would violate our "Permanence" principle: we include the number of steps of integration in the model definition. In fact, if attempted in a circular ring code using symplectic integration, variable time steps can lead to diffusion by destroying the linear invariants of the motion.

Notice that the validity of our model is ultimately determined by global concepts, but the description is still local. If the locality and the permanence principle are respected, we will show in section 2.4 how the blocks can be freely moved and put together to construct a circular ring just like the LEGO blocks used by children.

\subsection{State Variables}

Function Dynamics In the previous subsection, we have assumed that the reader is already familiar with the 6-vector $\boldsymbol{x}$. In Eq.(1), the transformation $\mathbf{f}$ describes the propagation of $\boldsymbol{x}$ through an element. 
It is possible to extend the dynamics of 6 -vectors to functions of the 6 -vector $x$. The function $\mathbf{f}$

$$
\boldsymbol{x} \stackrel{f}{\rightarrow} \mathbf{f}(\boldsymbol{x})
$$

induces a functional map $T_{\mathbf{f}}$

$$
g_{\text {in }} \stackrel{T_{\mathbf{f}}}{\longrightarrow} g_{\text {out }},
$$

which acts on a function $g_{\text {in }}$ to make another function $g_{\text {out }}$ as follows:

$$
g_{\text {out }}=T_{\mathrm{f}}\left[g_{\text {in }}\right] \Longleftrightarrow g_{\text {out }}(\boldsymbol{x})=\left(g_{\text {in }} \circ f\right)(\boldsymbol{x}) \equiv g_{\text {in }}(f(x))
$$

This extension of the 6-vector dynamics to functions seems entirely formal and therefore completely useless. Indeed, by definition, if you know the transformation $\mathbf{f}$, you automatically know how to evolve functions using Eq.(4). However, this is the small price one must pay in order to use Lie methods in the perturbative techniques used in the analysis of the one-turn map. Lie operators act on functions and therefore they are objects which "live" in the same space as $T_{\mathrm{f}}$.

For electron machine, there is a non-trivial extension of the 6-vector dynamics whose purpose is to compute the effect of diffusion and damping on a Gaussian distribution. It is the envelope dynamics which we now discuss.

Local Barycentre-Envelope Dynamics In the absence of photon emission (i.e. if the radiation is classical), the propagation of a particle distribution through one block is entirely determined by the operator $T_{\mathrm{f}-1}$ acting on the distribution function because the map is deterministic. The latte: includes radiation with $\hbar=0$ or the usual Hamiltonian dynamics.

$$
\psi_{\text {out }}=\frac{T_{\mathbf{f}-1}\left[\psi_{\text {in }}\right]}{\left|\mathbf{f}^{-1}\right|}, \quad|\mathbf{f}|=\operatorname{det}\left(\frac{\partial f_{i}}{\partial x_{j}}\right) .
$$

The determinant $\left|\mathbf{f}^{-1}\right|$ insures the preservation of the total probability (or number of particles). Its value is one for a symplectic map.

Our goal is to find the equivalent of Eq.(5) in the presence of diffusion. In the case of stochastic diffusion, what is possible to compute correctly is the evolution of a Gaussian distribution function (under certain approximations which will become clear later) ${ }^{4}$.

We start with a Gaussian distribution defined at the entrance face of an element:

$$
\psi(\boldsymbol{x})=\frac{1}{(2 \pi)^{n} \sqrt{\operatorname{det} \Sigma}} \exp \left[-\frac{1}{2} \Sigma_{i j}^{-1}(x-\bar{x})_{i}(x-\bar{x})_{j}\right],
$$

where $n=(1,2$ or 3$)$ denotes the degree of freedom. The various parameters in the Gaussian have an immediate statistical intepretation. The barycentre $\overline{\boldsymbol{x}}$ is the average of $\boldsymbol{x}$ over the distribution:

$$
\overline{\boldsymbol{x}}=\langle\boldsymbol{x}\rangle=\int \boldsymbol{x} \psi(\boldsymbol{x}) d \boldsymbol{x} .
$$

\footnotetext{
${ }^{4}$ In practice, this is not possible for a general distribution $\psi_{i n}(\mathbf{x})$. We can write an equation for $\psi_{\text {out }}$ only formaly.
} 
The envelope is a matrix

$$
\Sigma_{i j}=\left\langle(x-\bar{x})_{i}(x-\bar{x})_{j}\right\rangle,
$$

whose components are also called the 2 -nd order moments.

To propagate this distribution through the element, we expand $f$ around the barycentre $\overline{\boldsymbol{x}}$ as follows:

$$
\mathbf{f}(\boldsymbol{x})=\mathbf{f}(\overline{\boldsymbol{x}}+(\boldsymbol{x}-\overline{\boldsymbol{x}}))=\mathbf{f}(\overline{\boldsymbol{x}})+M(\boldsymbol{x}-\overline{\boldsymbol{x}}) \ldots
$$

The linear map induced by the matrix transformation $M(x-\bar{x})$ leaves the distribution of Eq.(6) as a Gaussian. Therefore only the barycentre and the envelope are changed. Following our discussion on function dynamics, we get

$$
\left.\begin{array}{l}
\overline{\boldsymbol{x}} \longrightarrow f(\overline{\boldsymbol{x}}) \\
\Sigma \longrightarrow M \Sigma M^{t}
\end{array}\right\}
$$

Now, it is possible to add a diffusion effect which can be represented by a matrix $B(\overline{\boldsymbol{x}})$. This matrix is entirely computable from the local dynamics of an element. The transformation which describes the propagation of $\Sigma$ through the block is given by

$$
\Sigma \longrightarrow M \Sigma M^{t}+B
$$

It is important to notice that $B$ as well as $M$ are local properties of the element. For example, an insertion of an additional element at some other place affects neither $M$ nor $B$ [1]. Therefore one can be very formal and simply say that the state variable contains the usual 6-vector with the induced deterministic function dynamics and, in addition, it contains the envelope $\Sigma$. In the case where the stochastic matrix $B$ vanishes the envelope $\Sigma$ is a redundant component of the state variable because it is equivalent to the dynamics of quadratic function under linear transformations.

However for this formal transformation to remain physically valid, the distribution $\psi$ must represent always a Gaussian whose size remains within the domain of validity of Eq.(7). Otherwise non-linearities become important and the approximation of Eq.(9) is no longer self-consistent. For example, the motion of the barycentre is not given by $f(\bar{x}) .^{5}$

Imagine you are standing on a floor where many LEGO blocks are scattered. You have examined already how each LEGO block works. Now it is play time! In the next section, you will connect these blocks.

\subsection{Connecting Local Blocks into a Ring}

If we connect a Bend with a Drift, the $x$-axis and $y$-axis of the Drift must match those of the Bend. Now, let us connect a Bend, a Drift and a Bend. Let us insist that the last bend be tilted vertically. Now we panic! We find a disagreement between $x-y$ coordinates at the exit of the Drift and those at the entrance of the vertical Bend. The LEGO blocks do not fit into one another. Solution: if you are a kid, you simply ask your parents to

\footnotetext{
${ }^{5}$ Note that if $f(x)$ is not linear, $\langle f(x)\rangle \neq f(\bar{x})$. See, for example, Ref.!18].
} 
buy a new type of block, a matching block, namely a $x$-y rotation of angle $\phi$ (here $\phi=90$ degrees). See Fig. 3,

Figure 3: A rotation is necessary in passing into the vertical Bend. In the lower figure, the connection between $D$ and a vertical Bend is shown. The solid (dashed) arrows are the $y(x)$ axes defined in each block.

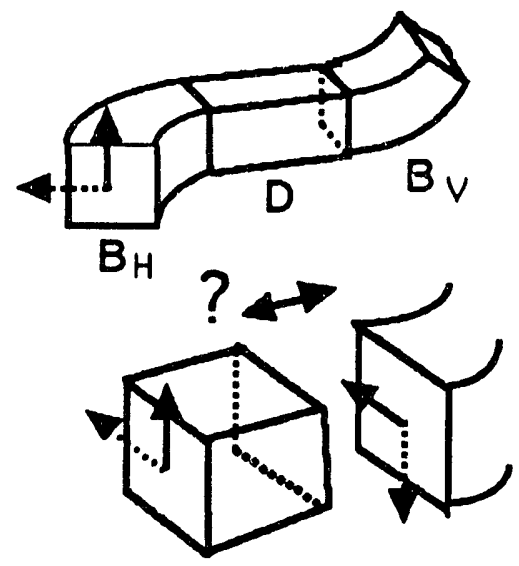

Thus the properly "glued" transformation for these three blocks is

$$
\mathbf{f}_{\text {total }}=\mathrm{f}_{B_{V}} \circ R(-\pi / 2) \circ \mathrm{f}_{D} \circ \mathrm{f}_{B_{H}}
$$

where

$$
\begin{array}{ll}
\mathrm{B}_{V}: & \boldsymbol{x}^{\prime}=\mathbf{f}_{B_{V}}(\boldsymbol{x}), \\
\text { Entrance of } \mathrm{B}_{V}: & \boldsymbol{x}^{\prime}=R(-\boldsymbol{\pi} / 2) \boldsymbol{x}, \\
\text { Drift : } & \boldsymbol{x}^{\prime}=\mathbf{f}_{D}(\boldsymbol{x}), \\
\mathrm{B}_{H}: & \boldsymbol{x}^{\prime}=\mathbf{f}_{B_{H}}(\boldsymbol{x}) .
\end{array}
$$

Here, $\mathrm{f}_{B_{V}}$, for example, represents the transformation for the vertical Bend. The introduction of the rotation $R$ is artificial, because the very choice of the coordinate system on the faces of the blocks is arbitrary. Of course, by defining the blocks as we did, the rotation between blocks is fixed by the relative orientation. It is important to point out that nothing unphysical is introduced by this transformation, it is just like changing the names of the axes.

What about the function dynamics and the envelope dynamics? The answer is immediate since a rotation in the $x-y$ plane is a 6 -vector transformation (leaving time and energy constant). Hence, it induces a functional map. For example, we have for our three blocks:

$$
T_{\mathbf{f}_{\text {total }}}=T_{\mathbf{f}_{B_{H}}} T_{\mathbf{f}_{D}} T_{R(-\pi / 2)} T_{\mathbf{f}_{B_{V}}}=T_{\mathbf{f}_{B_{V}} \circ R(-\pi / 2) \circ \mathbf{f}_{D} \circ \mathbf{f}_{B_{H}}}
$$

Then the equivalent of Eq.(12) for the envelope dynamics is

$$
\Sigma_{\text {out }}=M_{3}\left(R(-\pi / 2)\left(M_{2}\left(M_{1} \Sigma_{\text {in }} M_{1}^{t}+B_{1}\right) M_{2}^{t}+B_{2}\right) R(-\pi / 2)^{t}\right) M_{3}^{t}+B_{3},
$$

where $M_{1}, M_{2}$ and $M_{3}$ are the linear maps of $\mathrm{f}_{B_{H}}, \mathrm{f}_{D}$ and $\mathrm{f}_{B_{V}}$ respectively as defined in Eq.(7). 
Remarks on Multi-Block Elements Some readers may believe that the shape of the design trajectory $(6$-vector $=0)$ through the element should be a reflection of the internal composition of the element. For example, one might think that horizontal wigglers could be viewed as a succession of horizontal Bends separated by $\pi$ rotations (or something even more horrible in the case of the helical wiggler). It is possible but very difficult to follow this line of thought. In reality, the geometry of the wiggler is very simply expressed in Cartesian coordinates [24], and therefore should be integrated in Cartesian coordinates [5]. This makes them Drift blocks as said in section 2.1. Hence they should not be multi-block elements.

On the other hand, bending magnets, depending on their geometry, can be made of up to 5 blocks internally. We outline this in appendix B.

Now, you have a complete set of LEGO blocks. We must close the ring.

Closing the Ring Following the prescription we just outlined, one keeps connecting Bends and Drifts one after another into the desired bizarre geometry. Finally, we reach the last block. The prescription is obvious. The origin of the exit face of the last block must coincide with the origin of the entrance face of the first block. The faces must be also parallel. And finally, last but not least, a rotation must be applied to line up the frames. We have closure.

We should emphasize again that the design trajectory $(6$-vector $=0$ ) is not necessarily a closed orbit ncr any realistic orbit. In fact, wigglers, certain types of combined function bends and realistic fringe fields will prevent the closure of the 0 vector even for the ideal machine. The so-called design orbit does not exist.

\section{Global System: One-Turn Map}

Now that the ring is made, we can produce the so-called "one-turn" map. You sit at an arbitrary point, called check-point. It is located at the entrance face of some arbitrary block (surface of section). There you will study how the state vector evolves turn after turn. All the details of the individual block construction and of their juxtaposition become irrelevant. The primary interest of the game has shifted. The central question is now: what happen to the state vectors, $\boldsymbol{x}, \psi(\boldsymbol{x}), \Sigma$, etc... upon iteration.

All the concepts which we have purposely ignored up to this point become relevant because they are consequences of the iteration procedure:

1. for the case of the deterministic dynamics

(a) closed orbit at $s=0$, (b) normal mode decomposition, (c) tunes, (d) betatron functions at $s=0$, (e) chromaticity, (f) resonances, (g) tune shifts, (h) dispersion, (i) shape of quasi-invariants, (j) short/mid/long term stability, etc.

2. for the case of the envelope dynamics (a) damping partition number, (b) equilibrium envelope (more accurate expression of the equilibrium emittances), (c) all that can be deduced from synchrotron radiation integrals. 
Now, what do we do? If these concepts are not derivable from some representation of the one-turn map we are in deep waters! But, of course, it is just the opposite. They are derivable from the $N$-jets representation of the one-turn map which is an approximation of the map $T_{\mathbf{f}}$.

We now give a quick overview of the "Hamiltonian-free" description of perturbation theory. An approximation of the one-turn map is defined in terms of $N$-jets in 3.1. The essence of the perturbation is clarified in 3.2. The approximated one-turn map is related to some familiar concepts in 3.3. The beam-envelope is discussed in a parallel fashion in 3.4. In 3.5 it is argued that the perturbation cannot replace the tracking. Some useful formulas for the response of the system to a small perturbation, such as the linear tune shift, is explained from our point of view in 3.6.

\subsection{Approximations of the One-turn Map: $N$-Jets}

The one-turn map gives $\boldsymbol{x}_{\text {out }}=\mathbf{f}\left(\boldsymbol{x}_{\text {in }}\right)$ for $\boldsymbol{x}_{\text {in }}$ and $\boldsymbol{x}_{\text {out }}$ defined at the check-point. This map contains almost all the necessary information of particle motion, as long as you stay always at the check-point. If you plot $\boldsymbol{x}$ every turn sitting at the check-point, the resulting graph is what we call the Poincare plot. This plot is obtained by using the one-turn map only.

It is useful to define a (period one) fixed point as

$$
x_{0}=f\left(x_{0}\right)
$$

This point (and its translation through the ring) is called a closed orbit. Caution: neither the uniqueness nor the stability of such a point is assured.

As we did with the barycentre, we express the one-turn map around the fixed point $\boldsymbol{x}_{0}$ by introducing $\boldsymbol{\xi}=\boldsymbol{x}-\boldsymbol{x}_{0}$ and changing $\mathbf{f}(\boldsymbol{x})$ as $\mathbf{f}(\boldsymbol{x})-\boldsymbol{x}_{0}$ :

$$
\boldsymbol{\xi}_{\text {out }}=\mathbf{f}\left(\boldsymbol{\xi}_{\text {in }}\right), \quad[\mathbf{f}(0)=0 .]
$$

From now on $\boldsymbol{\xi}$ is just a dummy ${ }^{6}$.

Dynamics of $N$-jets Now, we will introduce a finite basis of our function dynamics in order to discuss the ordinary perturbation theory.

An arbitrary function $g^{(N)}$ of $\xi$ is called $N$-jets if it is expressed in terms of the monomial functions restricted to a given degree $N$ :

$$
g^{(N)}=\sum_{|\mathbf{m}|=0}^{|\mathbf{m}|=N} G_{\mathbf{m}}|\mathbf{m}\rangle,
$$

where $|\mathbf{m}\rangle$ is the monomial function such that, when applied to $\boldsymbol{\xi}$, it makes $\boldsymbol{\xi}^{\mathbf{m}}$ :

$$
|\mathrm{m}\rangle(\boldsymbol{\xi})=\xi^{\mathrm{m}} \equiv \xi_{1}^{m_{1}} \xi_{2}^{m_{2}} \xi_{3}^{m_{3}} \xi_{4}^{m_{4}} \xi_{5}^{m_{3}} \xi_{6}{ }^{m_{6}} \text {. }
$$

\footnotetext{
${ }^{6}$ From now on we employ so-called "active view". In treating a transformation, the active view transforms the object whereas the passive view changes the reference frame. The relation between active and passive views is similar to that between Heisenberg and Shrödinger pictures in quantum mechanics.
} 
Here, $\mathbf{m}=\left(m_{1}, \cdots, m_{6}\right)$ is a multi-index of 6 positive integers. The norm is the usual "taxi driver norm" (i.e. $|\mathbf{m}|=\sum m_{i}$ ). Notice that we did already introduce a quadratic restriction in the envelope calculation $(|\mathbf{m}| \leq 2)$. Indeed, the transformation of the Gaussian distribution in section 2.3 is almost equivalent to the transformation of the polynomial $\Sigma_{i j} \xi_{i} \xi_{j}$ where $\Sigma_{i j}$ is a vector of 21 independent coefficients. We revisit this in section 3.4 .

The $N$-jets, i.e. the truncated expansion Eq.(15), is another kind of state vector. This truncation induces a map $T_{\mathrm{f}}^{(N)}$ from $T_{\mathrm{f}}$ which acts on the $N$-jets and make another $N$-jets.

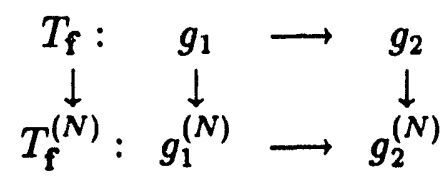

That is, $T_{\mathrm{f}}^{(N)}[g]$ is $T_{\mathrm{f}}[q]$ less terms of an order higher than $N$. The dynamics on functions with automatic truncation at a degree $N$, thus induced, is often called the dynamics of $N$-jets. Thus, we have

$$
T_{\mathbf{f}}^{(N)}\left[g^{(N)}\right]=g^{\prime(N)}=\sum_{|\mathbf{m}|=0}^{|\mathbf{m}|=N} G_{\mathbf{m}} T_{\mathbf{f}}|\mathbf{m}\rangle
$$

Here use was made of the linear property ${ }^{7}$ of $T_{\mathbf{f}}$. The function $T_{\mathbf{f}}^{(N)}|\mathrm{m}\rangle$ can be expanded in the monomial basis:

$$
T_{\mathbf{f}}|\mathbf{m}\rangle=\sum_{|\mathbf{n}|=0}^{|\mathbf{n}|=N} \tilde{M}_{\mathbf{n m}}|\mathbf{n}\rangle
$$

When substituted into Eq.(17), it induces

$$
T_{\mathbf{f}}^{(N)}\left[g^{(N)}\right]=g^{\prime(N)} \equiv \sum_{|\mathbf{n}|=0}^{|\mathbf{n}|=N} G_{\mathbf{n}}^{\prime}|\mathbf{n}\rangle, \quad G_{\mathbf{n}}^{\prime}=\sum_{|\mathbf{m}|=0}^{|\mathbf{m}|=N} \tilde{M}_{\mathbf{n m}} G_{\mathbf{m}} .
$$

From Eq.(19), we see that the functional one-turn map $T_{f}^{(N)}$ has a matrix representation given by $\tilde{M}_{\mathrm{nm}}$. This implies that we can study the properties of $T_{\mathrm{f}}$ in a closed manner.

It can be said that the physical content (or the lack of physical content) of classical perturbation theory is determined entirely by the truncation mechanism of the one-turn

${ }^{7}$ The functional operator $T_{\mathrm{f}}$ and $T_{\mathrm{f}}^{(N)}$ are linear:

$$
T_{\mathrm{f}}\left[\sum_{i} \alpha_{i} g_{i}\right]=\sum_{i} \alpha_{i} T_{\mathrm{f}}\left[g_{i}\right]
$$

Proof

$$
T_{\mathbf{f}}\left[\sum_{i} \alpha_{i} g_{i}\right](x)=\left[\left(\sum_{i} \alpha_{i} g_{i}\right) \circ \mathbf{f}\right](x)=\sum_{i}^{n} \alpha_{i}\left(g_{i} \circ \mathbf{f}\right)(x)=\sum_{i} \alpha_{i} g_{i}(\mathbf{f}(x))=\sum_{i} \alpha_{i} T_{\mathbf{f}}\left[g_{i}\right](x) .
$$


map. ${ }^{8}$ The jet dynamics depicted above, is convenient because it gives us the usual nonlinear perturbation theory (Deprit or Birkhoff style). Also, the jets are easily computed: there exist modern computer techniques such as the so-called "differential algebra package" of Berz which permits the automatic computation of the monomial restriction directly from the block dynamics of the 6-vector we described above. ( If you write a "FORTRAN code" to push around the 6-vector, then such a package will permit the simultaneous computation of the monomial dynamics.)

We now ask the fundamental question: what can we compute out of $N$-jets? This is the purpose of the next section.

\subsection{Global Dynamics of $N$-jets: the Paradox}

We discuss the nature of the perturbation theory. Perturbation theory is a method to find approximate invariants of the motion and to express global properties in terms of these invariants.

Following a notation popularized by Dragt, let us denote by $\mathcal{M}^{(N)}$ the one-turn map induced on the $N$-jets by the functional map $T_{\mathrm{f}}^{(N)}$.

Question: for the symplectic map, does the following functional equation have a sciution

$$
T_{\mathrm{f}}[g]=g ?
$$

Note that the solution $g(\xi)$, if it exists, is an invariant of the motion.

Answer: in general NO. It is well known that the motion in an accelerator is nonintegrable. Therefore Eq.(20) cannot have a non-trivial ${ }^{9}$ solution (except for some trivial cases. A ring without a cavity, for example. Here, the energy is conserved hence it is a solution).

Now what about the same equation restricted to the $N$-jets: do we have a function $g^{(N)}=\sum_{|\mathbf{m}|=0}^{|\mathbf{m}|=N} G_{\mathbf{m}} \mid \mathrm{m}>$ which is a solution of the equation

$$
\mathcal{M}^{(N)}\left[g^{(N)}\right]=g^{(N)} \leftrightarrow \sum_{\mathbf{m}} \tilde{M}_{\mathbf{n m}} G_{\mathbf{m}}=G_{\mathbf{n}} ?
$$

The answer is: in general YES. The quadratic part of $\tilde{M}$ can be diagonalized exactly (Courant-Snyder invariants). The nonlinear extension of the Courant-Snyder invariants can be obtained from $\tilde{M}$ because it is nearly lower triangular when the vector $\mathbf{G}$ is ordered by increasing degree [14]. They are some subtleties when the linear tunes are on a resonance $\mathbf{m} \cdot \boldsymbol{\nu}=k ; k=$ integer. It is much easier to see all of this in terms of Lie transformations than in terms of the matrix $\tilde{M}$. The reader should be patient until the next subsection.

Clearly, the solution of Eq.(21) would be the Taylor series expansion of the solution of Eq.(20) to order $N$, if it existed. But we just said that Eq.(20) has no solution: what

\footnotetext{
${ }^{8}$ We choose a monomial expansion truncated at a finite degree $N$. There exist other truncation schemes which involve a totally different way of approximating the map. For example, in the work of Warnock and Ruth [21] on "fitted" maps, the approximation scheme is valid on tori fur from the origin.

${ }^{9}$ Constant functions are always solutions, but they don't teach us anything
} 
in the world is going on? The answer is simple: if the jets of higher and higher order are considered, i.e. when $N \rightarrow \infty$, the expression of Eq.(21) must diverge. The series is asymptotic.

Similar questions can be asked about the non-symplectic deterministic dynamics of the $N$-jets. For example, is there some function $g$ such that

$$
T_{\mathrm{f}}[g]=e^{-2 \alpha} g ?
$$

Here $\alpha$ is a positive number. In this case $g$ is an emmitance-like quantity which shrinks with a constant rate towards the origin. The parameter $\alpha$ can be viewed as a measure of the deviation from the symplectic condition. Again, the answer is the same: Eq.(22) has no globally defined solution, but the equation

$$
\mathcal{M}^{(N)}\left[g^{(N)}\right]=e^{-2 \alpha} g^{(N)}
$$

does.

So, in conclusion, if the dynamics of the $N$-jets leads to diverging expression what is it good for? The answer is subtle. It is discussed in the next section.

\subsection{So, where are my Global Goodies? Answer: Normal Forms}

Lie Transform To help in the discussion of the Global Goodies, it is useful to introduce Lie operators. In the symplectic case, this gives us some sort of pseudo-Hamiltonian for the one turn map and, in the non-symplectic case, it guaranties that whatever we do on the map, it stays near the symplectic group. 10

First, we introduce the usual functional derivative $\partial_{k}$. It is simply defined as follows

$$
\left(\partial_{k} g\right)(\xi) \equiv \lim _{\epsilon \rightarrow 0} \frac{g\left(\xi_{1}, \ldots, \xi_{k}+\epsilon, \ldots, \xi_{6}\right)-g(\xi)}{\epsilon}
$$

Using Eq.(24), we can define the Poisson bracket of two functions $g$ and $h$ :

$$
[g, h] \stackrel{\text { def }}{\equiv} \partial_{i} g J_{i j} \partial_{j} h
$$

Here, $J$ is the matrix

$$
J=\left(\begin{array}{cccccc}
0 & 1 & 0 & 0 & 0 & 0 \\
-1 & 0 & 0 & 0 & 0 & 0 \\
0 & 0 & 0 & 1 & 0 & 0 \\
0 & 0 & -1 & 0 & 0 & 0 \\
0 & 0 & 0 & 0 & 0 & 1 \\
0 & 0 & 0 & 0 & -1 & 0
\end{array}\right)
$$

\footnotetext{
${ }^{10} \mathrm{This}$ is of course a trivial definition. But it is written here to remind the reader that expressions such as $\partial_{k} f(g(\xi))$ do not have any meaning. If you are serious about using Lie methods and understanding maps such as $T_{\mathrm{f}}$, keep the notation clear in complex calculations. Otherwise, go straight to the bar, because, even sober, you will obtain psychedelic results.
} 
We are now in a position to define Lie operators and their associated functional map. Consider a function $h$; the Lie operator : $h:$ associated with $h$ (another Dragt notation, if you don't like it replace by $L_{h}$ or whatever you want) is defined by its action on an arbitrary function $g$ :

$$
: h: g \stackrel{\text { def }}{\equiv}[h, g] \text {. }
$$

The Lie map is just:

$$
\exp (: h:) g \stackrel{\text { def }}{\equiv} \sum_{k=0}^{\infty} \frac{: h:^{k}}{k !} g
$$

In Eq.(27) and Eq.(28), since we consid $u$ r the case where $h$ and $g$ are $N$-jets the question of convergence is then trivial ${ }^{11}$.

Notice that the Lie operator and its associated Lie map are objects of the same nature as the functional one-turn map. In fact, the restricted map $\mathcal{M}^{(N)}$ can be expressed as a product of Lie maps (Dra 3 t-Finn factorization[23]):

$$
\mathcal{M}^{(N)}=\mathcal{M}_{\text {linear }} \exp \left(: h_{3}:\right) \exp \left(: h_{4}:\right) \ldots \exp \left(: h_{N+1}:\right)
$$

This factorization is, however, of no direct help in understanding the bizarre equations of section 4.2 (Eq.(21) for example). For this we must introduce a normal (diagonal) representation of $\mathcal{M}$.

Normal Form First, let us define the action functions $J_{k}$ :

$$
\begin{gathered}
\mathbf{J}=\left(J_{1}, J_{2}, J_{3}\right), \\
J_{k}(\xi)=\frac{\xi_{2 k-1}^{2}+\xi_{2 k}^{2}}{2}, k=1,2,3 .
\end{gathered}
$$

Now, consider an arbitrary function of the actions $h(\mathbf{J})$. The reader can verify that $\exp (: h(\mathbf{J}):$ ) induces on the 6-vector (i.e. first order monomials) a J-dependent rotation. The angles of this rotation (tune shifts $\times 2 \pi$ ) are given by

$$
\mu_{k}=-\partial_{J_{k}} h(\mathbf{J})
$$

Furthermore, the actions $J_{k}$ are trivial invariants of our map. Of course, as we did for the $\operatorname{map} T_{\mathbf{f}}^{(N)}$, it is possible to find a matrix representation for $\exp (: h(\mathbf{J}):)$ :

$$
\exp (: h(\mathbf{J}):)|\mathbf{m}\rangle=\sum_{|\mathbf{n}|=0}^{|\mathbf{n}|=N} \tilde{R}_{\mathbf{n m}}|\mathbf{n}\rangle \Longleftrightarrow G_{\mathbf{m}} \longrightarrow \sum_{|\mathbf{n}|=0}^{|\mathbf{n}|=N} \tilde{R}_{\mathbf{m n}} G_{\mathbf{n}}
$$

Question: Is it possible for the linearly stable one-turn map $\mathcal{M}^{(N)}$ to be written as follows:

$$
\text { Normal Form } \mathcal{M}^{(N)}=\mathcal{A}^{-1} \exp (: h(\mathbf{J}):) \mathcal{A} \leftrightarrow \tilde{M}=\tilde{A}^{-1} \tilde{R} \tilde{A} ?
$$

\footnotetext{
${ }^{11}$ Not completely trivial because of the linear and quadratic part of $: h:$. It is left as an exercise for the reader.
} 
(Note that $\tilde{A}$ is the matrix representation of $\mathcal{A}$ as defined in Eq.(18).) Answer: in general yes for $N$-jets, except on a set of measure zero, which we call the resonant maps. The jet is resonant if, for the linear tune $\nu$, the following is true: $\mathrm{m} \cdot \boldsymbol{\nu}=k ; k=$ integer.

Remarkably, Eq.(34) contains the solution of Eq.(21) as well as all your other "Global Goodies". For example, the quantities $\mathcal{A}^{-1} J_{k}$ are immediate and obvious invariants.

The map $\exp (: h(\mathbf{J}):)$ describes motion on circles of radius $2 J_{k}=\epsilon_{k}$ where $\epsilon_{k}$ is the $k^{\text {th }}$ "invariant". From this it is clear that the map $\mathcal{A}$ describes deviation from the circles. It contains all the linear lattice functions (beta and similar things in the presence of coupling, etc...) as well as the so-called nonlinear distortion functions.

There is also another important case. Sometimes we consider a ring without an cavity. In this case, the normal form is different. One needs to refine the third action as

$$
J_{3}=- \text { Energy }=\xi_{6}
$$

In this case, the map $\exp (: h(J):)$ is a rotation in the planes 1 and 2 , and it is drifting in plane 3. The action of $\exp (: h(\mathbf{J}):)$ on the time of flight $\xi_{5}$ is given by:

$$
\Delta \xi_{5}=-\partial_{J_{3}} h(\mathbf{J})
$$

Needless to say, quantities like the linear momentum compaction $\alpha_{1}$ and its nonlinear counterparts are trivially read from Eq.(36). One also gets the amplitude dependent time of flight because Eq.(36) depends on $J_{1}$ and $J_{2}$. In this case, the dispersion (linear and nonlinear) is also a joke to extract from $\mathcal{A} .^{12}$

\subsection{Equilibrium Beam Envelope}

As we pointed out before, the non-symplectic dynamics (due to radiation) can be extended in a non-trivial way in the presence of stochastic diffusion.

When the beam is constrained to a linear region around the orbit of interest, we can restrict our calculation to linear maps. In addition, in the case of linear motion the equilibrium distribution, $\psi_{\infty}$, can be calculated. Thanks to the central limit theorem, we can restrict $\psi$ to a Gaussian

$$
\psi(\xi)=\frac{1}{(2 \pi)^{n} \sqrt{\operatorname{det} \Sigma}} \exp \left[-\frac{1}{2} \Sigma_{i j}^{-1} \xi_{i} \xi_{j}\right] .
$$

The one-turn map of the envelope can be written as

$$
\Sigma_{\text {out }}=M \Sigma_{\text {in }} M^{t}+B_{\text {one-turn }},
$$

${ }^{12}$ The dispersion is obtained as follows:

$$
D_{i}=\left(\mathcal{A}^{-1} \Xi_{i}\right) \circ \boldsymbol{X}_{6},
$$

where $\left.\left.\Xi_{1} \equiv \begin{array}{llllll}1 & 0 & 0 & 0 & 0 & 0\end{array}\right), \quad \Xi_{2} \equiv \begin{array}{llllll}0 & 1 & 0 & 0 & 0 & 0\end{array}\right), \cdots$ and $X_{6}$ is a function such that

$$
\forall \xi X_{6}(\xi)=\left(0,0,0,0,0, \xi_{6}\right)
$$

When applied to $\xi, D_{i}(\xi)$ gives i-th component of the closed trajectory with constant energy $\xi_{6}$, from which the (linear and nonlinear) dispersion function can be deduced. 
where $M$ and $B_{\text {one-turn }}$ are the transfer matrix (including radiation damping) and the diffusion matrix for a single turn, constructed by a repeated application of Eq.(13). From $M$, we can deduce the tunes and damping coefficients in terms of its eigenvalues. The equilibrium envelope (at the check point) $\Sigma_{\infty}$ is the envelope which is left invariant by Eq.(37).

The equilibrium envelope $\Sigma_{\infty}$ can be expressed as the solution of

$$
\Sigma_{\infty}=M \Sigma_{\infty} M^{t}+B_{\text {one-turn }}
$$

which in vector form can be rewritten as

$$
\hat{\Sigma}_{\infty}=\tilde{M}^{t} \hat{\Sigma}_{\infty}+\hat{B}_{\text {one-turn }}
$$

where $\hat{\Sigma}$ is a vector made from a matrix $\Sigma$ :

$$
\hat{\Sigma}=\left(\Sigma_{11}, \Sigma_{12}, \cdots\right)^{t},
$$

and $\tilde{M}$ is made from a matrix $M$ in such a way that ${ }^{13}$

$$
\tilde{M}^{t} \hat{\Sigma}=\left(\widehat{M \Sigma M^{t}}\right)
$$

Thus $\Sigma_{\infty}$ is expressed in a concise manner as $[1,3]$

$$
\hat{\Sigma}_{\infty}=\frac{1}{1-\hat{M}^{k}} \hat{B}_{\text {one-turn }} .
$$

This is how SAD [16] calculates the equilibrium envelope ${ }^{14}$. This gives us the beam sizes in terms of the physical coordinates at the check-point. For example, $\sqrt{\Sigma_{11}}$ is the horizontal beam size, which implicitly includes all the effects of the betatron functions, emittances, dispersion and energy spreads in a fashion more accurate than that given by the synchrotron integrals.

In the absence of damping and diffusion, there exist infinitely many invariant distributions: we regain the results of the previous section:

$$
\sigma_{k}^{-2} \mathcal{A}^{-1}\left[2 J_{k}\right]=\Sigma_{i j}^{-1} \xi_{i} \xi_{j}
$$

where the $\sigma_{i}^{2}$ 's are three arbitrary constants and $\mathcal{A}^{-1}$ is the operator of Eq.(34) in the case of 2-jets. The last expression expresses the invariants in terms of $\Sigma$ for the symplectic case. When damping is on but diffusion is still off, it is possible to diagonalize $\tilde{M}$ by a quasi-symplectic map ${ }^{15} \mathcal{A}^{-1}$ such that

$$
\mathcal{M} \mathcal{A}^{-1} J_{k}=\exp \left(-2 \alpha_{k}\right) \mathcal{A}^{-1} J_{k}
$$

\footnotetext{
${ }^{13}$ The transformation properties of $\Sigma_{i j}$ are dual the monomial $\xi_{i} \xi_{j}$, therefore they transform according to Eq.(18) with $N=2$, but with the transposed matrix. This is why we use the same notation for the matrix $\left(\hat{M}^{t}\right)$.

${ }^{14}$ It is to be understood that the closed orbit of $\bar{x}$ is found and $M$ is evaluated with respect to it.

${ }^{15}$ In this case, the diagonalized map is a rotation nultiplied by

$$
\exp \left[-2 \times \operatorname{diag}\left(\alpha_{1}, \alpha_{1}, \alpha_{2}, \alpha_{2}, \alpha_{3}, \alpha_{3}\right)\right] .
$$
}

The symplectic part of $\mathcal{A}^{-1}$ is in general different from Eq.(41). 
Here, $\mathcal{M}$ is the functional jet ${ }^{16}$ whose matrix is $\tilde{M}$.

The real question, with diffusion, is what happens to Eq.(39) if we transforn. it using $\tilde{A}^{t-1}=\left(\tilde{A}^{t}\right)^{-1}$

$$
\begin{gathered}
\tilde{A}^{t-1} \hat{\Sigma}_{\infty}=\left(\tilde{A}^{t-1} \tilde{M} \tilde{A}^{t}\right) \tilde{A}^{t-1} \hat{\Sigma}_{\infty}+\tilde{A}^{t-1} \hat{B}_{\text {one-turn }} \\
\hat{\Sigma}_{\infty}^{n e w}=\tilde{M}_{\text {new }}^{t} \hat{\Sigma}_{\infty}^{\text {new }}+\hat{B}_{\text {one-turn }}^{\text {new }} ?
\end{gathered}
$$

If, by some miracle, $\hat{B}_{\text {one-turn }}^{\text {ew }}$ has components only along the 3 functions $J_{k}$, then one can compute 3 integrals describing the vector $\hat{B}_{\text {one-turn }}^{\text {new }}$ and they can be interpreted as the fluctuations of the "emittances" 17. In general, it is not true that $\hat{B}_{\text {one-turn }}^{\text {new }}$ has projections only along the $J_{k}$ 's, it has components of order $\alpha_{k}$ along the other 18 directions[2]. Therefore, the synchrotron integrals are only approximate representations.

We recommend our method of calculation of the beam envelope as the primary numerical method to get the beam sizes because it does not rely on any ill-defined quantities. Of course, it is not forbidden (it is even recommended) to look at synchrotron integrals as a design guide. The validity of the latter is limited to $\alpha_{s} \ll 1$ in the case of 6 -d synchrotron integrals[28] and to $\nu_{s}, \alpha_{s} \ll 1$ for the case of 4 -d integrals[27].

\subsection{WARNING about Perturbation Theory}

We have outlined the origin of perturbation theory. An infinite dimensional functional equation is approximately solved by using a finite dimensional approximation of the functional map, $T_{\mathrm{f}}^{(N)}$. The results are, in general, the beginning of an asymptotic series.

You should never trust the results of such calculation without careful checks. Always compare your results with the underlying tracking code. There are all sorts of reasons why the perturbation series could be ill-behaved at even small amplitudes. For example, we mentioned that the diagonalization of the map $\mathcal{M}$ in Eq.(34) is always possible except for a set of measure zero corresponding to resonances. However, in reality, it suffices for the map $\mathcal{M}$ to be in the neighbourhood of a resonance $(\mathbf{m} \cdot \boldsymbol{\nu}=\mathbf{k})$ to greatly affect the usefulness of Eq.(34) in the study of the real map ${ }^{18}$.

The moral of the story stays the same: double check the results. This also applies to the non-symplectic map and the computation of the beam envelope. (Imagine a case where $\Sigma_{\infty}$ is too large for Eq.(7) to be applied.)

\subsection{The Issue of Useful Formulas}

In accelerator physics there are a myriad of useful formulas which express the dependence of a global quantity in terms of some other global properties and/or magnet strengths.

\footnotetext{
${ }^{16}$ Here $\mathcal{M}$ is $\mathcal{M}^{(2)}$. The superscript will be omitted hereafter whenever the meaning is clear.

${ }^{17}$ This approximation is used in Ref.[28].

${ }^{18}$ There are of course techniques to swim around resonances, but they are beyond the scope of this paper. They include single resonance normal forms [8] and redefinitions of the linear part of the map to move away from the resonance [9] as well as the use of totally different basis functions for the approximate map [21]. They even include combinations of the standard monomial expansion with a different basis [22].
} 
These formulas are derived under some idealized situation and rarely carried beyond the first order in perturbation. A typical and useful example is the expression of the change of the linear tune due to a quadrupole perturbation:

$$
\delta \nu= \pm \oint \frac{k(s) \beta_{ \pm}}{4 \pi} d s
$$

where \pm refers to the horizontal $(+)$ and the vertical $(-)$ planes.

Many experienced accelerator physicists who have tried, to their credit, to understand the concept of "Hamiltonian-free" perturbation theory are often left wondering about its usefulness in deriving cute little formulas.

Ugly DA-Formulas: Pluses and Minuses It should be clear to the reader that "ugly" formulas are obtainable according to our discussion: if one invest a little time to understand tools such as the "differential algebra" package of Berz, one realizes that it is possible to grind out big Taylor series map. One can also put system parameters in the Taylor series (magnet strengths, length, etc..). All the calculations and methods presented above follow by extending the 6 -vector to a $\left(6+N_{p a r}\right)$-vector where $N_{p a r}$ is the number of system parameters in the map. Any global quantity (beta functions, for example) is available from the perturbation, based on the one-turn map, and it can be propagated around the ring from point 1 to point 2 using the map from 1 to 2 .

What are the advantages and disadvantages of "DA" formulas? On one hand, the main advantage is absolutely clear: it is bug free. If you implement a new element in your code following our local LEGO block technique and you "DA-fied" the code, you will get all the global "goodies" as a function of this new element with a extremely low probability of mistake if some standard checks are performed at the original programming stage. There exist even "DA+Compiler" based software which allow you to produce these complex new maps in an almost interactive manner ${ }^{19}$. This advantage of the Taylor series approach allows you to compute a global quantity for your friend, which normally you do not care about, within minutes. On the other hand, the disadvantages are two-fold:

1. conceptual: you get just a formula with a pile of numerical coefficients. The global quantity is not written as a function of other global concepts so it does not teach you much.

2. computational: you may want to have the dependence of the quantity on some vast array of parameters. In this case, the number of monomials in a Taylor series of the $N$-jets goes like $\left(N+6+N_{\text {par }}\right) ! /\left[\left(6+N_{\text {par }}\right) ! N !\right]$. For large $N_{p a r}$ and moderate $N$ this number can get catastrophically big. The extraction of the Taylor series is slowed down, it may even grind to a halt because of memory problems.

One does not get "cute" formulas out of the Taylor series map. This is to be contrasted with the formula of Eq.(44): simple and works even with a trillion quadrupole insertions. In such cases an analytic formula may be more appropriate if one intends to use it over and over again.

${ }^{19}$ COSY-INFINITY,COSY-EXTERMINATOR/TRACY2,TLIE, etc... 
Cute formulas So we are back to the original question asked to us by many colleagues: yes, if I learn your techniques, I will grind out Taylor series and perform the extraction of global objects (beta, dispersion,etc...) more reliably than anybody else, but what about purely analytical formulas?

Here, we emphatically answer this worry by re-iterating what was said in the introduction: our technique is more powerful computationally as well as conceptually. Therefore the computation of formulas in terms of unperturbed global concepts is easier and cleaner with a map based theory. We will only say that a calculation of order " $n$ " in some smallness parameter $\epsilon$, will require the examination of a ring made out of " $n$ " maps with " $n$ " perturbative insertions. The maps are transformed into unperturbed normal variables by $\mathcal{A}^{0}$. The " $n$ " maps become rotations (phase advances). The Lie operator : $\epsilon V_{i}(\xi):$ of the $i^{\text {th }}$ insertion becomes : $\epsilon\left(\mathcal{A}_{i}^{0} V_{i}\right)(\xi)$ :. The calculation then proceeds order by order in $\epsilon$. The presence of $\mathcal{A}^{0}$ in the perturbation insures that everything is expressed in terms of the unperturbed global concepts ${ }^{20}$. Notice that $\mathcal{A}^{0}$ which is an input to the "cute" formulas, is computed using the $\mathrm{N}$-jets formalism and your favorite "DA-fied" tracking code. For a general theoretical framework, the reader must consult Ref. [6], and Ref. [7] for a complicated 2nd order example.

Finally, we make a comment on the radiation integrals. They expresses the equilibrium beam sizes in terms of integrations of betatron functions, dispersion and so on. On the other hand, the beam size has a firm physical meaning while the dispersion is a limiting concept. Imagine that we insert a thin quadrupole magnet at the check-point and we want to calculate the change of the beam sizes. If the insertion is centered around the closed orbit, for example, all we should do is just replace Eq.(38) by

$$
\Sigma_{\infty}=K M \Sigma_{\infty}(K M)^{t}+B_{\text {one-turn }}
$$

where $K$ represents the matrix of the inserted magnet. We can easily evaluate the effect on the beam sizes accurately by use of Eq.(40) with $M$ replaced by $K M$. On the other hand, if one uses the radiation integrals, one should evaluate the effect on the betatron functions, dispersions etc all throughout the ring, ending up with approximate results. This is a detour and a quite indirect way to do this job. As we said before, the radiation integrals are useful and concise formulas which we can use for rough estimates. This is, however, not the definition of the beam sizes.

\footnotetext{
${ }^{20}$ Note that $\epsilon\left(\mathcal{A}_{i}^{0} V_{i}\right)(\xi)$ is simply $\epsilon V_{i}\left(\xi^{\prime}\right)$ where $\xi^{\prime}$ is obtained by a coordinate transformation associated with $\mathcal{A}^{0}$. For example, in the presence of mid-plane symmetry, the derivation of Eq.(44) requires for $\xi^{\prime}$ :

$$
\begin{aligned}
\xi_{1}^{\prime} & =\sqrt{\beta_{x}} \xi_{1} \\
\xi_{2}^{\prime} & =\frac{1}{\sqrt{\beta_{x}}} \xi_{2}-\frac{\alpha_{x}}{\sqrt{\beta_{x}}} \xi_{1} \\
\xi_{3}^{\prime} & =\sqrt{\beta_{y} \xi_{3}} \\
\xi_{4}^{\prime} & =\frac{1}{\sqrt{\beta_{y}}} \xi_{4}-\frac{\alpha_{y}}{\sqrt{\beta_{y}}} \xi_{3} .
\end{aligned}
$$
}

This transformation is just the usual Courant-Snyder transformation. 


\section{Summary}

We have looked over what we call the "modern approach", which can be summarized as follows:

1. The one-turn map (plus partial transfer maps) is the fundamental object, which should be regarded as the nature itself, even though something is different from our real world.

2. To construct the one-turn map, you should not rely on the global, theoretical, and/or prejudiced concepts (Locality Principle). All approximations used in constructing it (i.e. models) should be kept fixed (Permanence Principle) within a single job (like the laws of nature).

3. In utilizing the one-turn map, you can introduce further approximations, provided they are defined clearly in terms of the one-turn map. Do not break the permanence principle at this point by replacing the laws. Do not go dangerously away from the limit of the approximations.

Let us clarify the above statements. We have already discussed the fundamental distinction between local and global concepts. We should also classify the global concepts into physical, limiting and fuzzy concepts:

1. physical concepts: one-turn map, transfer map, and quantities derivable from them directly. For example,
(a) closed orbit,
(b) tunes and equilibrium beam sizes $\left\langle x^{2}\right\rangle_{\infty}$, when the one-turn map is linear,
(c) dispersion for coasting proton beam, etc.

They are both measurable and touchable.

2. limiting concepts: those which are derived from the one-turn map with approximation. Many of them would be physical concepts of a different system with different models (i.e. different physics laws). For example,

(a) dispersions in the presence of a cavity. It becomes physical concept in the limit of a coasting proton beam.

(b) "equilibrium emittances". In electron rings, such objects do not exist as invariants. The limiting world is the asymptopia $\alpha \longrightarrow 0$.

(c) Courant-Snyder "invariants" becomes physical in the limits of linear mapping and no-radiation.

3. fuzzy concepts: all ill-defined concepts like most political slogans. Logically there is no need to discuss these because they are products of misunderstanding. An example: sometimes people seem to talk on the phase advances between two different point, without defining them explicitly. In fact, several different ways of factorizing the transfer matrix are known. The phase advance and $\beta$ differ for different factorization schemes. Thus without additional clear definitions, they are fuzzy concepts. This misunderstanding may come from applying 2-d concepts to 4-d or 6-d cases. 
By definition, all the local concepts are "physical".

The dangers of fuzzy concepts are clear. It is a crisis of physics.

The danger of limiting concepts exists when equating two of them. The equation may be exact only in the limiting case. For example, the maximum x-excursion of a particle and the Courant-Snyder invariant are related through $\sqrt{\beta}$. In the limiting case of linear motion and no-radiation, both are clear physical concepts. The invariant is essentially the area of the ellipse described by the particle. In the presence of radiation, however, buth become unphysical: the amplitude should be regarded as a stochastic variable and only the beam size $\left\langle x^{2}\right\rangle$ is a physical concept. Finally, in the presence of nonlinearities, in particular in the chaotic regions, the concept of the beam size is itself falling apart, then in that limit, only the one-turn map remains physical.

So, be careful and always check the validity of using the limiting concepts by checking with the one-turn map ( $\simeq$ tracking) and with your brain!

Remember that what you are really interested in should be physical concepts.

This will help you in using limiting concepts correctly when the usual approximations break down.

Acknowledgement One of the authors (E. F.) thanks the Foundation for High Energy Accelerator Science for financial support and Dr. S. Kurokawa for his gracious hospitality. He also acknowledges financial support from United States Department of Energy under Contract No. DE-AC03-76SF00098.

\section{A Fibre Bundle Structure of LEGO Loop}

Our way of making one-turn maps can be clearly explained in terms of the terminology of fibre bundles [17].

A fibre bundle $B$ is defined as a combination $B=(S, Y, G)$ :

1. $S$ is a manifold (base space).

2. $Y$ is a topological space. Each point $s \in S$ is associated with a topological space $Y_{s}$ (fibre). All $Y$, are equivalent to $Y$ in the topological sense.

3. A group $G$ acts on $Y$, that is, $G: Y \rightarrow Y$.

In the accelerator dynamics context, the base space is the loop of the ring, so that $S=S^{1}$ (one-sphere). The fibre $Y$ is the state vectors, such as

1. the coordinate system $\left(\mathbf{e}_{x}, \mathbf{e}_{y}\right) . G$ is rotation $R(\phi)$.

2. the 6 -vector $\boldsymbol{x} . G$ is the symplectic group and represented by $\mathrm{f}$.

3. function $g$. $G$ is the functional map representation of the symplectic group: i.e. $T_{\mathrm{f}}$.

4. $N$-jets $g^{(N)}=\sum_{|\mathbf{m}| \leq N} G_{\mathbf{m}}|\mathbf{m}\rangle . G$ is a restriction $T_{\mathbf{f}}^{(N)}$ of $T_{\mathrm{f}}$.

5. distribution function $\psi(\boldsymbol{x})$.

6. barycentre and envelope, $(\bar{x}, \Sigma)$. ( $G$ is the almost symplectic transformation $) n$ the barycentre and an almost symplectic affine transformation on the envelope: $\left.M \Sigma M^{t}+B\right)$. 
For illustration, we use the 6-vector $\boldsymbol{x}$ as an example.

The base space $S$ has a set of neibourhoods $U_{i}$. In accelerator, $U_{i}$ is an open interval $\left(s_{1}, s_{2}\right)$. Locally $B$ is a direct product

$$
B \simeq Y \times U_{i}, \quad \text { (locally) }
$$

A map $\pi: B \rightarrow S$ is called the projection. The fibre at $s, Y_{s}$ is defined by $Y_{s} \equiv \pi^{-1}(s)$. A map $u: Y \times U_{i} \rightarrow Y$ determines a particular value of the coordinate of $b \in\left(Y_{s}, s\right)$. Two different choices of coordinates $x=u(b)$ and $x=u^{\prime}(b)$ are related by $G$. The point $b$ is a physical quantity but its coordinates are arbitrary. Let us call this 'gauge' degree of freedom. By choosing one gauge $u_{i}$ in each $Y \times U_{i}, B$ is endowed with a coordinate system.

If $s \in U \cap U^{\prime}$, where $U$ and $U^{\prime}$ are neighbourhoods of $S$, a point $b \in Y \times\left(U_{i} \cap U_{j}\right)$ are still given two coordinates, $\left(\boldsymbol{x}_{i}, s\right)$ and $\left(\boldsymbol{x}_{j}, s\right)$. They are related to each other by $g_{i j} \in G$ :

$$
u_{i}(b)=g_{i j} \circ u_{j}(b), \text { or } \boldsymbol{x}_{i}=g_{i j} \circ \boldsymbol{x}_{j} .
$$

Here $g_{i j}$ does not depend on $b$. This $g_{i j}$ corresponds to our third (matching) block defined in 2.4.

Thus $B$ can be imagined as a series of shect of papers aligned to each other according to some prescribed rule. (See Fig. 4). In some cases, by choosing a gauge properly, all

Figure 4: Fibre bundle with a prescribed transformation rule $g_{i j}$. The same point in $B$ can have different coordinates in different neighbourhoods. They are related by a gauge transformation $g_{i j} \in G$. The curve shows the closed orbit (in case $Y$ is a 6 -vector), or the equilibrium beam size ( in case $Y$ is an envelope). A continuous curve (continuous in $B$ ) can be written as $y=f(s)$ in the fibre bundle sense. When expressed by the local coordinates, it has discontinuity at the borders of neighbourhoods.
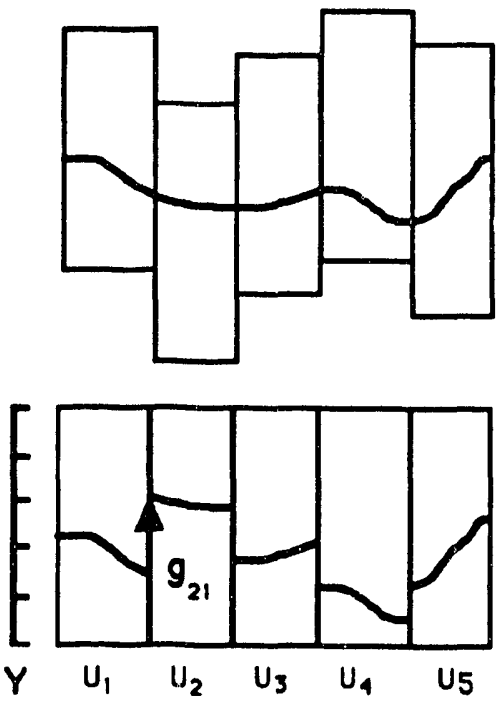

$g_{i j}$ can be made 1 , the identity of $G$. In this case, the bundle is said trivial. In general, however, bundle is not trivial.

Thus, we can define a continuous curve in $B$, which in general appears discontinuous when seen in terms of the coordinates as numbers. We can express the curve as $y=f(s)$ with the fibre bundle convention: that is, $y=y_{j}$ in $U_{j}$ and it jumps to $g_{i j} y_{j}$ when going from $U_{i}$ to $U_{j}$. Similarly, the particle equation of motion can be written as

$$
\frac{d x}{d s}=[x, H], \quad \text { in Fiber Bundle sense. }
$$


In each $U_{i}, \boldsymbol{x}$ and $H(x)$ are expressed arbitrarily within the gauge freedom. We have stressed in the main text that we should choose the most convenient gauge for constructing the map.

A typical case of nontrivial fibre bundle occurs when some vertical bends exist in a certain way. See Fig. 5. Here you cannot close your ring without using a gauge

Figure 5: Nontrivial fibre bundle. The point a is the check-point. You go through the LEGO ring. In this case, you refuse to use the matching block. Thus, you should use some rotated coordinate frame in some blocks. This corresponds to choosing a frame of reference by a parallel transport. In the end, however, you find that you are obliged to use at least one matching block in order to close the ring.

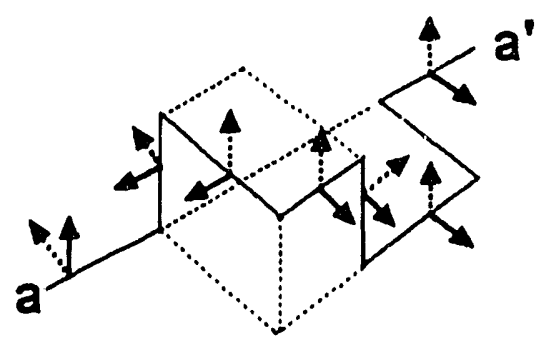

transformation at least once. The necessary amount of rotation $\phi$ in this case is intrinsic to the loop of the ring. Thus, we have proved that in general, it is impossible to define a globally continuous coordinate frame without torsion. ${ }^{21}$

\section{B Rectangular Bend}

Here, we will illustrate an art of building a model. In particular, we will show that an element is not always composed of the blocks one would naively guess. Our discussion is based on two seemingly similar elements: the shifted quadrupole bend and the slice "donut" bend. They both have quadrupole components and can be called "combined function bending magnets".

The simplest one to discuss is the shifted quadrupole. It is also the strangest. Such a magnet is obtained by simply sliding a quadrupole. The resulting bend is a combined function bend with a perfectly rectangular geometry. Notice that the orbit through the bend cannot be an arc of circle unless the gradient is zero. How can we take advantage of the simplicity of the Cartesian geometry. Look at Fig. 6 and follow our words. As a LEGO block, this element is a bend with the entrance face labeled " 1 " and the exit face labeled " 4 ". As such, we give a map from " 1 " to " 4 ". It can be moved and rotated anywhere in the ring in accordance with the LEGO block formalism. Now, what about the inside? To take advantage of the Cartesian symmetry, we do as follows:

1. Bring $x$ from 1 to 2 , using a polar coordinate ("rotation" around the y-axis). The Hamiltonian for this is that of the drift (free) space but expressed in polar coordinates. Notice that this "rotation" is a bend block of zero thickness.

\footnotetext{
${ }^{21}$ To be accurate, it should be said that you can choose always a planner circle for the coordinate frame no matter how the equation of motion becomes complicated. This is possible, but extremely difficult. There is no need to do such a laborious and fruitless job.
} 
Figure 6: Geometry of a rectangular parallel face combined function bend (=shifted quadrupole). Since the magnetic field in the body (from 3 to 4) has a translational symmetry in the direction of the dotted line, the internal coordinate frame should be the rectangular one, even if the Hamiltonian has magnetic fields. On the other hand, from 1 to 2 , the coordi-

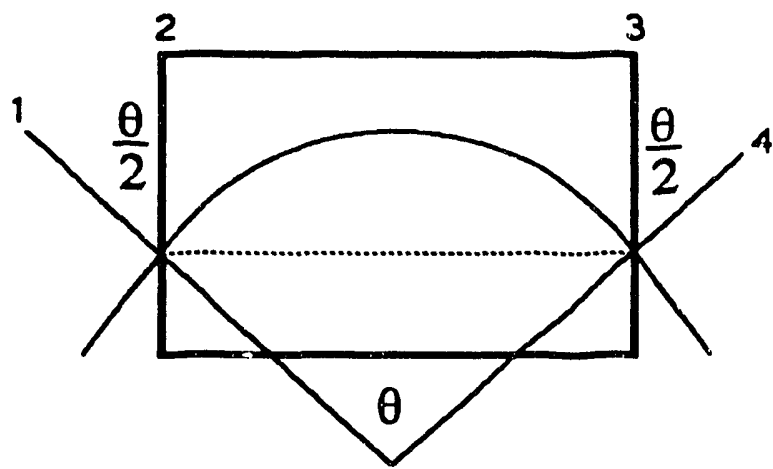
nate frame should be the polar one, whereas the Hamiltonian is that of a drift space.

2. At the face 2, the coordinates are connected continuously. (We do not need the matching block).

3. Then we can put some fringe effects if we want in frame 2 . That would be a drift block (ignore if it confuses you).

4. Now, the most important piece, we propagate through the magnet from face 2 to 3 in Cartesian coordinates using the Hamiltonian:

$$
H=-\sqrt{x_{6}^{2}-x_{2}^{2}-x_{4}^{2}}+\frac{q B_{0} x_{1}}{p_{0}}+\frac{K}{2}\left(x_{1}^{2}-x_{3}^{2}\right) .
$$

This propagation is a Drift according to our nomenclature!

5. Then we tag another fringe field at 3 and we "rotate" to 4.

So, the entire element is a "rotation", a fringe, a bend in Cartesian, another fringe and a final "rotation". In the block language, you have a bend, 3 drifts and a bend. From the point of view of the trajectory, it is the central drift that does all the bending!

Before going on with the next example, a word is needed on the y-axis "rotation". This rotation mixes the direction of propagation (z-axis) and the transverse $\mathrm{x}$-axis. Therefore it involves the Hamiltonian of the system (here a drift space). In the next example, the same rotation will involve the bend Hamiltonian. If this confuses you, think of translating a quadrupole along the z-axis. This must involve two drifts along this axis. If the quadrupole is immersed in a solenoid field, then it would involved propagation forward and backward in the solenoid medium. See reference [11] for more details.

Now, what about the sliced "donut"? L.ook at Fig. 7. Think of it as a donut with current winding around it with the pipe and the windings cut a funny angle. It is clear that in the centre of this object there is an almost perfect invariance of the Hamiltonian if we move along an arc of circle. (Rotational invariance). Near the faces it might be easier to use a Cartesian frame perpendicular to the faces. So the magnet is gotten by the following blocks: 
Figure 7: Geometry of a cylindrical parallel face bend. Since the magnetic field has a rotational symmetry, it is natural to express the Hamiltonian of the body, 3 to 4 , in polar coordinates.

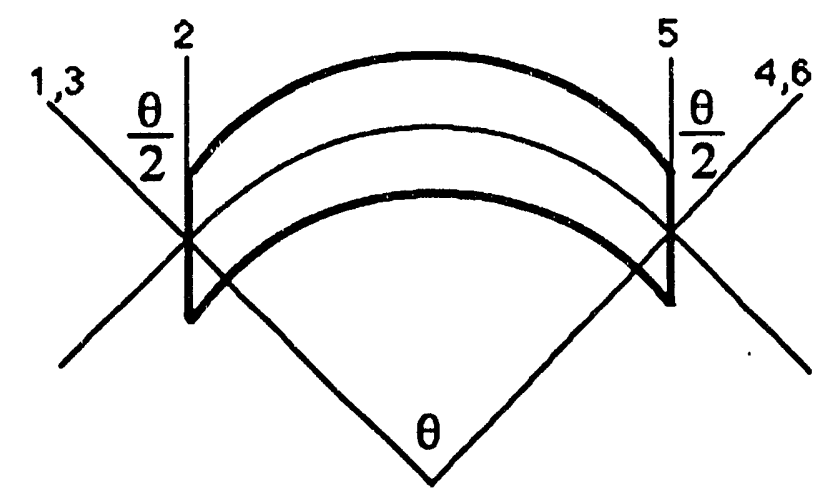

1. A "rotation" in free space from 1 to 2 ,

2. A fringe effect at 2 ,

3. "Rotation" in the bend medium back to 3. (This time the Hamiltonian is that with the magnet field, even if no field exist for $x>0$.)

4. Integration of the bend all the way from 3 to 4 using cylindrical coordinates and the Hamiltonian

$$
H=-\left(1+\frac{x_{1}}{\rho}\right) \sqrt{x_{6}^{2}-x_{2}^{2}-x_{4}^{2}}+\frac{q B_{0} x_{1}}{p_{0}}+\frac{q B_{0} x_{1}^{2}}{2 p_{0} \rho}+\frac{K}{2}\left(x_{1}^{2}-x_{3}^{2}\right)+K O\left(\frac{1}{\rho}\right) .
$$

5. Then "rotate" in the bend medium back to 5 where you put a fringe effect.

6. Finally, "rotate" in free space all the way to 6.

The total block is a bend, a drift, 3 bends, a drift and a final bend. In Eq.(48), the term $K O(1 / \rho)$ is generated by Maxwell's equations in curvilinear coordinates. The quantity $\rho$ is the radius around which the coordinates are expressed in the cylindrical frame.

With these two examples we have illustrated that even though each element is a bend from the LEGO point of view and must be treated identically when moved around the lattice, in practice, Maxwell's equations will tell you what it should be like inside the element. This is extremely important in the proper treatment of small machines [11].

\section{References}

\section{Papers written in the spirit of this paper}

[1] K. Hirata and F. Ruggiero, Treatment of Radiation in Electron Storage Rings, CERN Report, LEP Note 611 (1988).

[2] K. Hirata and F. Ruggiero, Part. Accel.28 (1990) 137. (Proceedings of XIV Int. Conf. on High Energ. Accel. 1989 Tsukuba.)

[3] K. Ohmi, K. Hirata, K. Oide, From Beam Envelope Matrix to Synchrotron Radiation Integrals, to be published.

[4] E. Forest and J. Milutinovic, Nucl. Instrum. Methods Phys. Res., A269 (1988) 474. 
[5] E. Forest and K. Ohmi, Symplectic Integration of Complex Wigglers, to appear as a KEK Report (1992).

[6] E. Forest, J. Mathematical Physics 31 (1990) 1133.

[7] J. Bengtsson and J. Irwin, Analytical Calculations of Smear and Tune Shift, SSC Technical Report, SSC-232, (1990).

[8] E. Forest and J. Irwin, Single Resonance Theory with Maps, Proceeding of the Workshop on Nonlinear Problems in Future Particle Accelerators, Capri, Italy, 1990, W. Scandale and G. Turchetti Editors, World Scientific Publishing Co., p. 46, (1991).

[9] K. LaMon, Lie series in an extended region of phase space, J. Phys. A: Math. Gen. 23 (1990) 3875.

[10] F. Ruggiero, E. Picasso and L. A. Radicati, Annals of Physics (N.Y.) 197 (1990) 396.

[11] E. Forest, The Absolute Bare Minimum for Tracking in Small Rings, to appear as a LBL report, submitted to Part. Accel.

[12] E. Forest, M. Berz and J. Irwin, Normal Form Methods For Complicated Periodic Systems: A Complete Solution Using Differential Algebra and Lie Operators. Part. Accel. 24 (1989) 91.

[13] R. L. Warnock, Close Approximation to Invariant Tori in Nonlinear Mechanics, Phys. Rev. Lett.166 (1991) 1803.

[14] A. Antillón, E. Forest, B. Hoeneisen and F. Leyvraz, Transport Matrices for Nonlinear Lattice Functions, Nucl. Instrum. Methods Phys. Res., A305 (1991) 247.

\section{Other useful papers}

[15] A. J. Dragt, in Physics of High Energy Accelerators, proceedings of the Summer School on High Energy Particle Accelerators, Fermilab, 1981, edited by R.A. Carrigan, I.R. Huson, and M. Month (AIP Conf. Proc. No. 87) (AIP, New York, 1982), p. 147.

Today, the Lie transformation becomes a kind of language that everybody should know to discuss beam dynamics. Essentially, this is nothing but a concise way of writing down a symplectic transformation. This text is very well written but it does not emphasize circular rings and consequently violates our locality principle. For example, it spends tim: explaining how to compute the Lie operator for a single magnet. According to our discussion, the Lie operator is only useful to the one turn map in circular rings. This text barely touches this issue. So one should be careful in reading it. We recommend to read it until Chap.5. The rest should be read after you become more familiar with the perturbation calculation.

[16] K. Hirata, An Introduction to SAD, Proc. 2-nd ICFA Beam Dynamics Workshop, CERN 88-04 (1988). A computer program SAD (Strategic Accelerator Design) has been developed in KEK since 1987.

[17] N. Steenrod, The Topology of Fibre Bundles, Princeton Univ. Press (1951).

[18] K. Hirata , Nucl. Instrum. Methods Phys. Res.A269 (1988) 7.

[19] H. Yoshida, Phys.Lett. A150 (1990) 262. Among all of references on symplectic integrators, in the best of our knowledge, this paper should be read first, because of its simplicity and clarity, although similar results have been obtained a little earlier by other author. See E. Forest and R.D. Ruth, Physica D 43, 105, (1990). 
[20] M. Berz, Differential Algebraic Description of Beam Dynamics to Very High Orders Part. Accel. 24 (1989) 109.

[21] R.L. Warnock and R.D. Ruth, Phys. Rev. Lett., 66 (1991) 990.

[22] J. S. Berg and R.L. Warnock, IEEE Particle Accelerator Conference, May 1991 p.1654, also available as SLAC-PUB-5458.

[23] A.J. Dragt and J.M. Finn, Lie series and invariant functions for analytic symplectic maps, J. Math. Phys., 17 (1976) 2215.

[24] K. Halbach, Physical and Optical Properties of Rare Earth Cobalt Magnets, Nucl. Instrum. Methods Phys. Res.187 (1981) 109.

[25] L. O. Chua and H. Kokubo, Normal Forms for Nonlinear Vector Fields - Part 1: Theory and Algorithm, IEEE Transactions on Circuits and Systems, 35 (1988) 863.

Papers very important but not to read until you become well acquainted with modern point of view

[26] E.D. Courant and H.S. Snyder, Ann. Phys. 3 (1958) 1.

[27] M. Sands, SLAC report, SLAC-121 (1970).

[28] A. Chao, J. Appl. Phys.,50 (1979) 595. 

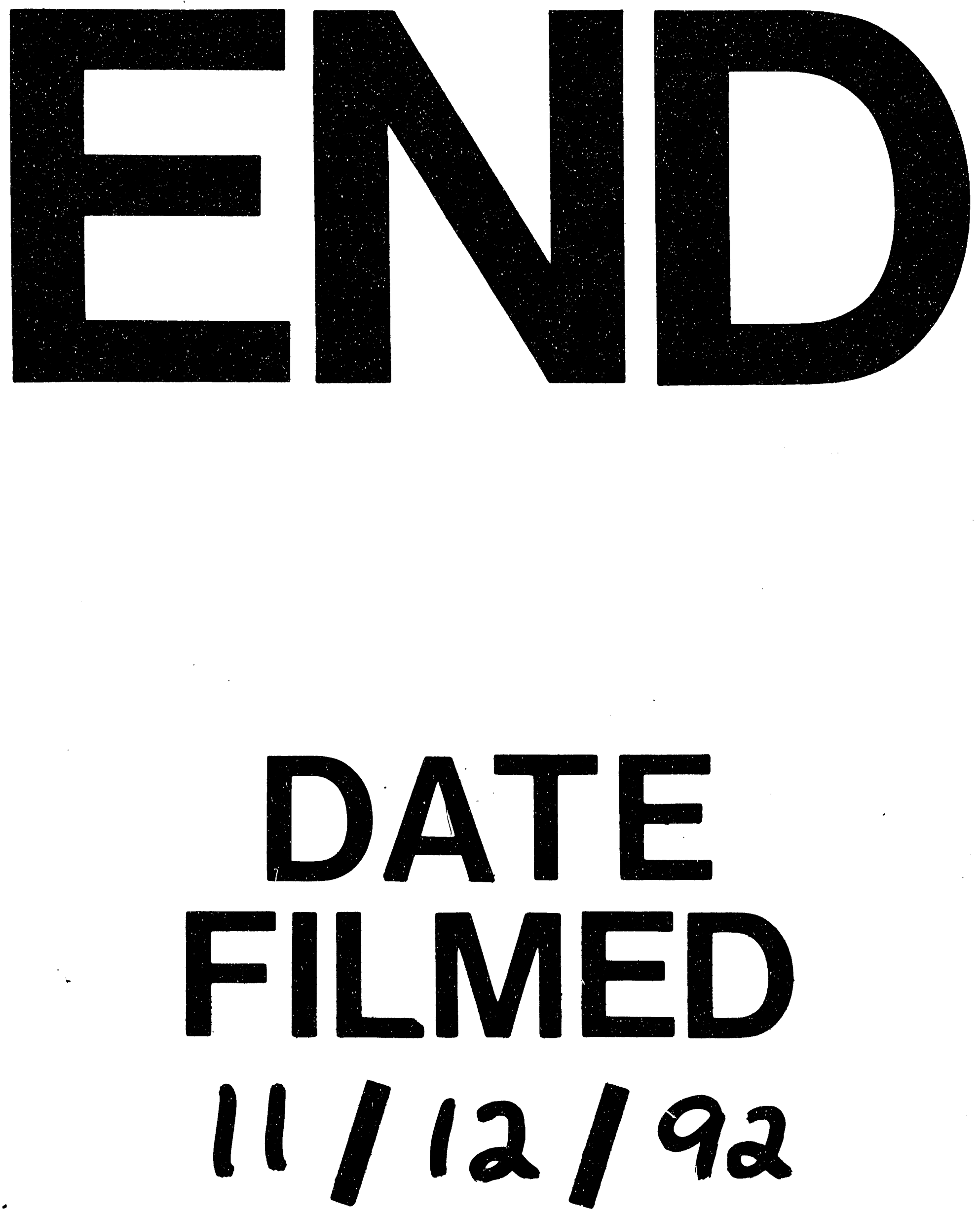

1 
